\title{
The nonrelativistic limit of (central-extended) Poincaré group and some consequences for quantum actualization
}

\author{
Juan S. Ardenghi, ${ }^{1, a)}$ M. Castagnino, ${ }^{1, b)}$ and R. Campoamor-Stursberg ${ }^{2, c)}$ \\ ${ }^{1}$ Instituto de Astronomía y Física del Espacio, CC67 suc. 26, 1428 Buenos Aires, \\ Argentina \\ ${ }^{2}$ I.M.I.-Universidad Complutense de Madrid, 3 Plaza de Ciencias, E-28040 Madrid, Spain \\ (Received 29 April 2009; accepted 2 September 2009; published online 15 October 2009)
}

\begin{abstract}
The nonrelativistic limit of the centrally extended Poincare group is considered and their consequences in the modal Hamiltonian interpretation of quantum mechanics are discussed [O. Lombardi and M. Castagnino, Stud. Hist. Philos. Mod. Phys 39, 380 (2008); J. Phys, Conf. Ser. 128, 012014 (2008)]. Through the assumption that in quantum field theory the Casimir operators of the Poincare group actualize, the nonrelativistic limit of the latter group yields to the actualization of the Casimir operators of the Galilei group, which is in agreement with the actualization rule of previous versions of modal Hamiltonian interpretation [Ardenghi et al., Found. Phys. (submitted)]. (C) 2009 American Institute of Physics.

[doi:10.1063/1.3243822]
\end{abstract}

\section{INTRODUCTION}

This paper is mainly devoted to find the nonrelativistic limit of an extended Poincare group. But as the motivation of this research was inspired by a new interpretation of quantum mechanics we begin this introduction discussing this subject.

For a long time after its first formulation, the interpretation of quantum mechanics was deeply tied to possible measurement outcomes. Due to some technical difficulties arising from this orthodox interpretation, new approaches intended to give a more realistic description were worked out, resulting in some interpretations that emphasize certain aspects or properties of quantum systems, and establishing sharply the limits of the corresponding approach.

In this sense, the modal interpretations first proposed by van Fraasen ${ }^{1}$ establish a succinct distinction between the quantum state and the value state of a system. While the former describes the possible physical properties of the system, the latter represents the properties that can actually be detected.

Clearly this leads to a probabilistic relation among these concepts, separated into two precise categories.

(1) The category of wave functions and density matrices, i.e., the world of probabilities and potential facts.

(2) The category of actual facts, i.e., facts appearing in real measurements. Examples are given when a dot appears in a photographic plate or a Geiger counter detects the presence of a particle (facts that are usually considered to be related with the quantum collapse).

In this context, the notion of "actualization" serves as the link between the two preceding classes. Actualization can be seen as the process in which a potential fact from (1) becomes an actual fact. Obviously this procedure is not free from some difficulties and constraints. The Kochen-Specker theorem ${ }^{2,3}$ specifies that not all the observables do actualize, but only some of

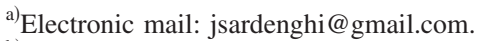

${ }^{b}$ Electronic address: ingatsac@iafe.uba.ar

${ }^{c)}$ Electronic address: rutwig@pdi.ucm.es
} 
them. The various interpretations of quantum mechanics are therefore committed to select a context in which the observables that will acquire a definite value are defined. Thus, for example, the Copenhagen interpretation establishes that only observables of the measurement apparatus actualize, while for De Broglie-Böhm interpretation the position observables actualize. The concept of actualization has therefore become a crucial tool to interpret quantum mechanics.

Enlarging the previous ansatz in Ref. 4 a new member of the family of quantum mechanics interpretations was introduced, the so-called modal Hamiltonian interpretation (MHI). In this case, the Hamiltonian of the quantum system plays a central role in the actualization problem. More specifically, it is postulated that the observables corresponding to the eigenbasis of a complete set of commuting observables containing the Hamiltonian $H$ and the constants of motion must actualize. This approach is therefore heavily based on the symmetry properties. In successive refinements of the MHI, it was proven that it agrees with the "Ithaca interpretation" of quantum mechanics proposed by Mermin in Ref. 6. We recall that Mermin's approach is based on various desiderata or necessary conditions that any reasonable interpretation of quantum mechanics should satisfy.

Following the lines of this MHI, it was further proven in Ref. 7 that for systems invariant under the Galilei group, the three Casimir operators of the central mass extended Galilei group, namely, the mass, the spin squared, and the internal energy $W$ do actualize. Finally, the existence of a kinetic component in the Hamiltonian $H$ was proven. Eliminating this fictitious component $W \rightarrow H$, the postulates of Ref. 4 were easily recovered.

In this paper we present a alternative version of the nonrelativistic limit of the centrally extended Poincaré group and its consequences in the interpretation problem. We will also consider the nonrelativistic limit of the (centrally extended) Poincare group as a necessary preparation to implement the MHI for quantum field theory (QFT). As this theory is essentially a scattering theory, we will focus mainly on the preparation and measurements stages. Nevertheless, we will consider the interaction stage, which has no direct experimental verification. Therefore, at this stage, our conclusions will be merely hypothetical. Several features of the problem are also considered.

Section II presents some known features concerning the extended Galilei and Poincaré groups, developing in Sec. III the nonrelativistic limit of the trivially extended Poincaré group in both the classical and group theoretical counterparts. In Sec. IV we analyze the behavior of the Casimir operators under this limit. In Sec. V we will consider the particle number operator. Then in Sec. V we will present our example: following the ideas of Ref. 7 we show that, if we postulate that in QFT the Casimir operators of the Poincare group actualize, then the nonrelativistic limit of the latter group yields the actualization of the Casimir operators of the Galilei group (namely, those that actualize according to the previous version of MHI of Ref. 7). In this section we will essentially consider the one particle case. Finally, in Sec. VI, we consider the particle number that will also actualize, in the preparation and measurement stages, since it labels different representations of the corresponding groups. This section introduces the many particle case. We will also discuss the difference between the in and out stages and the interaction one and we will discuss some features of an eventual version of MHI for QFT, introduce the preparation and measurement apparatuses in detail, and we will consider also the corresponding actualization. Section VII contains our conclusions.

\section{THE (EXTENDED) GALILEI AND POINCARÉ GROUPS}

In what follows, we will use the kinematical bases for both the Galilei and Poincaré groups, as well as their extensions.

For the generators of the Galilei group we take the operators $H, P_{i}, J_{i}, K_{G i}$, where the last ones correspond to the Galilean boosts. In this basis, the commutation relations are given by

$$
\left[P_{i}, P_{j}\right]=0, \quad\left[J_{i}, J_{j}\right]=i \varepsilon_{i j k} J_{k}, \quad\left[J_{i}, P_{j}\right]=i \varepsilon_{i j k} P_{k}, \quad\left[H, P_{i}\right]=0, \quad\left[H, J_{i}\right]=0,
$$




$$
\left[K_{G i}, K_{G j}\right]=0, \quad\left[J_{i}, K_{G j}\right]=i \varepsilon_{i j k} K_{G k}, \quad\left[H, K_{G i}\right]=-i P_{i}, \quad\left[P_{i}, K_{G j}\right]=0,
$$

where $i, j, k, \ldots=1,2,3$ and $i=\sqrt{-1}$. As is well known, this group admits a nontrivial central extension by a central charge $M$ that commutes with the generators of the Galilei group. The brackets of the extension are those of (1), with the exception of $\left[P_{i}, K_{G j}\right]=0$, that is replaced by

$$
\left[P_{i}, K_{G j}\right]=-i \delta_{i j} M .
$$

While for an ordinary presentation (or at the classical level) this extension is unnecessary, for quantum representations with an arbitrary phase (i.e., such that $|\phi\rangle \sim \exp (i \omega)|\phi\rangle)$ this central extension is unavoidable (Refs. 8 and 9, Chap. 3). Then the extended group is the product of the primitive Galilei group by a boolean group with generator $M$.

The generators of the Poincare group are taken as $H, P_{i}, J_{i}, K_{P i}$, where the last ones are the Lorentz boosts. The commutation relations of the Poincaré group can be formulated in fourdimension Lorentz space as

$$
\begin{gathered}
{\left[P_{\mu}, P_{\nu}\right]=0, \quad\left[M_{\mu \nu}, P_{\rho}\right]=i\left(\eta_{\mu \rho} P_{\nu}-\eta_{\nu \rho} P_{\mu}\right),} \\
{\left[M_{\mu \nu}, M_{\rho \sigma}\right]=i\left(\eta_{\mu \rho} M_{\nu \sigma}-\eta_{\mu \sigma} M_{\nu \rho}-\eta_{\nu \sigma} M_{\mu \rho}+\eta_{\nu \rho} M_{\mu \sigma}\right),}
\end{gathered}
$$

where $\mu, \nu, \ldots=0,1,2,3$ and

$$
P_{\mu}=\left(H, P_{i}\right), \quad M_{\mu \nu}=\left(\begin{array}{cc}
0 & K_{P i} \\
-K_{P i} & J_{i j}
\end{array}\right),
$$

$\eta_{\mu \nu}$ being the metric tensor of space-time, $J_{k}=\frac{1}{2} \varepsilon_{k i j} J_{i j}$, and therefore $M_{\mu \nu}$ is defined by $\left(K_{P i}, J_{k}\right)$. Then Eqs. (3) can be rewritten as follows:

$$
\begin{gathered}
{\left[P_{i}, P_{j}\right]=0, \quad\left[J_{i}, J_{j}\right]=i \varepsilon_{i j k} J_{k}, \quad\left[J_{i}, P_{j}\right]=i \varepsilon_{i j k} P_{k}, \quad\left[H, P_{i}\right]=0, \quad\left[H, J_{i}\right]=0,} \\
{\left[K_{P i}, K_{P j}\right]=-i \varepsilon_{i j k} J_{k}, \quad\left[J_{i}, K_{P j}\right]=i \varepsilon_{i j k} K_{P k}, \quad\left[H, K_{P i}\right]=-i P_{i}, \quad\left[P_{i}, K_{P j}\right]=-i \delta_{i j} H .}
\end{gathered}
$$

It follows at once from (1) and (4) that the Poincaré and Galilei groups share a splittable seven-dimensional subgroup $I S O(3) \times\langle H\rangle$ generated by $H, P_{i}, J_{i}$, and having commutators

$$
\left[P_{i}, P_{j}\right]=0, \quad\left[J_{i}, J_{j}\right]=i \varepsilon_{i j k} J_{k}, \quad\left[J_{i}, P_{j}\right]=i \varepsilon_{i j k} P_{k}, \quad\left[H, P_{i}\right]=0, \quad\left[H, J_{i}\right]=0,
$$

where $i, j, k, \ldots=1,2,3 .^{10}$

\section{EXTENDED GALILEI GROUP AS A LIMIT OF THE POINCARÉ GROUP}

It is well known that the Galilei group can be recovered from the Poincare group by means of an Inönü-Wigner contraction. ${ }^{11}$ It is therefore natural to ask whether such a situation can be generalized to the centrally extended Galilei group, which is one of the relevant objects in quantum mechanics. However, since the Poincaré group does not admit nontrivial central extensions, ${ }^{12}$ the situation is more involved. To solve this problem, we will consider two limiting processes: the first using the classical reasoning of special relativity, and the second through a special InönüWigner contraction from a trivial extension of the Poincare group. ${ }^{13}$

\section{A. The classical nonrelativistic limit}

We perform the first approach using the nonrelativistic limit in its usual way. Let the position be given by $x^{\mu}=\left(c t, x^{i}\right)$, the absolute velocity be $U^{\mu}=\left(c, v^{i}\right)$, the absolute time be $\tau=\left[t^{2}-\left(1 / c^{2}\right)\right.$ $\left.\times\left(x^{2}+y^{2}+z^{2}\right)\right]^{1 / 2}$, the absolute mass be $m_{0}$, and the relative mass be $m=\gamma m_{0}$, where $\gamma=(1$ $\left.-\beta^{2}\right)^{-1 / 2}$ and $\beta=v / c$. Then, if $p^{i}=m v^{i}$, the absolute momentum reads $P^{\mu}=\left(E / c, p^{i}\right)$, where the energy is given by $E=c^{2} m=c^{2} \gamma m_{0}$. We obtain the nonrelativistic limit for either $v \rightarrow 0$ or $c \rightarrow \infty$, namely, if $\beta^{2} \rightarrow 0$. Expanding $\gamma$ into powers of $\beta$, we get 


$$
\gamma=\left(1-\beta^{2}\right)^{-1 / 2}=1+\frac{1}{2} \beta^{2}+o\left(\beta^{2}\right) .
$$

If we neglect the quadratic term $o\left(\beta^{2}\right)$ below, i.e., physically we are considering velocities that are small compared to the velocity of light, then

$$
P^{\mu}=\left(\frac{E}{c}, p^{i}\right)=\left(c \gamma m_{0}, \gamma m_{0} v^{i}\right)=\left(1+\frac{1}{2} \beta^{2}\right)\left(c m_{0}, P_{G}^{i}\right)=\left(c m_{0}+\frac{c}{2} \beta^{2} m_{0}, P_{G}^{i}+\frac{1}{2} \beta^{2} P_{G}^{i}\right),
$$

where $P_{G}^{i}=m_{0} v^{i}$ is the nonrelativistic momentum (the $P_{i}$ of the Galilei group). Now the term $\mathrm{cm}_{0}$ can be promoted to an operator, precisely the central charge $c M$. Taking into account the nonrelativistic limit $\beta^{2} \rightarrow 0$, we deduce that

$$
\frac{E}{c}=\frac{H}{c}=c m=c m_{0}+\frac{c}{2} \beta^{2} m_{0}=c M+\frac{T}{c} \rightarrow c M,
$$

where $T=\frac{1}{2} m_{0} v^{2}$ is the classical kinetic energy. Taking the normalization $c=1$ (as in the commutation relations) in the limit $\beta \rightarrow 0$, the latter expression reduces to

$$
H \rightarrow M \text {. }
$$

Then (2) is the nonrelativistic limit of $\left[P_{i}, K_{P_{j}}\right]=-i \delta_{i j} H$.

Let us finally observe that, from Eq. (7), the proper mass $m_{0}$ can be considered, in the one particle case that we are considering (see Sec. VI for the many particles case), as the internal energy $W$, since it is the energy at the center of mass system.

Moreover, it follows from Eq. (6) for $\beta \rightarrow 0$ that $p^{i}=P_{P}^{i} \rightarrow P_{G}^{i}$, i.e., the relativistic Poincaré momenta go to the nonrelativistic Galilei momenta. Finally, as the Lorentz boost is given by

$$
x^{\prime}=\gamma(x+v t), \quad t^{\prime}=\gamma\left(t+\beta_{c} \frac{x}{c}\right)
$$

and, in the $\beta \rightarrow 0$ limit, this Galilei boost reads ${ }^{14}$

$$
x^{\prime}=x+v t, \quad t^{\prime}=t .
$$

Using these equations, it is easy to deduce that the relativistic boosts do not commute, while the nonrelativistic ones do. In this sense, $\left[K_{G_{i}}, K_{G_{j}}\right]=0$ is the nonrelativistic limit of the commutator $\left[K_{G_{i}}, K_{G_{j}}\right]=-i \varepsilon_{i j k} J_{k}$. This means that the Galilei group is the nonrelativistic limit of the Poincare group.

\section{B. Trivially extended Poincaré group and the generalized Inönü-Wigner contraction}

In this paragraph we show that the previous nonrelativistic limit can also be justified by pure group theoretical arguments. For this purpose, we reorder the generators of the Poincare algebra $I S O(1,3)$ in the following way:

$$
\begin{gathered}
{\left[J_{i}, J_{j}\right]=i \varepsilon_{i j k} J_{k}, \quad\left[J_{i}, K_{P j}\right]=i \varepsilon_{i j k} K_{P k}, \quad\left[J_{i}, P_{j}\right]=i \varepsilon_{i j k} P_{k}, \quad\left[H, J_{i}\right]=0,} \\
{\left[P_{i}, P_{j}\right]=0, \quad\left[K_{P i}, K_{P j}\right]=-i \varepsilon_{i j k} J_{P k}, \quad\left[H, K_{P i}\right]=-i P_{i}, \quad\left[P_{i}, K_{P j}\right]=-i \delta_{i j} H, \quad\left[H, P_{i}\right]=0,}
\end{gathered}
$$

where in the left hand side of the first line we have listed all the commutator with $J_{i}$, which are related to the space isotropy. We now extend the group trivially, i.e., in such a way that all the generators of (8) commute with a trivial central charge $M$. Then we have a new algebra $I^{M} S O(1,3)=I S O(1,3) \times\langle M\rangle$ with basis $\left\{J_{i}, P_{i}, K_{P i}, H, M\right\}$. We perform the following change of the generators' basis: 


$$
\bar{H}=H-M \text {. }
$$

Over the new basis $\left\{J_{i}, P_{i}, K_{P i}, \bar{H}, M\right\}$, all commutators of (8) remain the same, with the only exception of

$$
\left[P_{i}, K_{P j}\right]=-i \delta_{i j} H=-i \delta_{i j}(\bar{H}+M) .
$$

Observe, in particular, that space isotropy is preserved. We claim that this algebra contracts naturally onto the centrally extended Galilei algebra given by (1) where the last commutator is replaced by (2). More specifically, the contraction,

$$
\operatorname{ISO}(1,3) \times\langle M\rangle \rightsquigarrow G(2) \times\langle M\rangle,
$$

is determined by the rescaling transformations (over the basis $\left\{J_{i}, P_{i}, K_{P i}, \bar{H}, M\right\}$ ) defined by

$$
J_{i}^{\prime}=J_{i}, \quad P_{i}^{\prime}=\varepsilon P_{i}, \quad K_{P i}^{\prime}=\varepsilon K_{P i}, \quad \bar{H}^{\prime}=\bar{H}, \quad M^{\prime}=\varepsilon^{2} M .
$$

It is straightforward to verify that the space isotropy remains unchanged by this change of basis. The remaining commutation relations change as follows:

$$
\left[P_{i}^{\prime}, P_{j}^{\prime}\right]=0, \quad\left[K_{P i}^{\prime}, K_{P j}^{\prime}\right]=-i \varepsilon^{2} \varepsilon_{i j k} J_{P k}^{\prime}, \quad\left[H, K_{P i}\right]=-i P_{i}^{\prime}, \quad\left[H^{\prime}, P_{i}^{\prime}\right]=0,
$$

and

$$
\left[P_{i}^{\prime}, K_{P j}^{\prime}\right]=-i \delta_{i j} \varepsilon^{2}(\bar{H}+M)=-i \delta_{i j}\left(\varepsilon^{2} \bar{H}^{\prime}+M^{\prime}\right) .
$$

For the limit $\varepsilon \rightarrow 0$, the nonvanishing commutators are

$$
\begin{gathered}
{\left[P_{i}^{\prime}, P_{j}^{\prime}\right]=0, \quad\left[K_{P i}^{\prime}, K_{P j}^{\prime}\right]=0, \quad\left[H^{\prime}, P_{i}^{\prime}\right]=0,} \\
{\left[H^{\prime}, K_{P i}^{\prime}\right]=-i P_{i}^{\prime}, \quad\left[P_{i}^{\prime}, K_{P j}^{\prime}\right]=-i \delta_{i j} M^{\prime} .}
\end{gathered}
$$

It follows at once that the contracted algebra is isomorphic to the extension of the Galilei algebra given by (1) where $\left[P_{i}, K_{G_{j}}\right]=0$ is replaced by (2).

As a consequence, the classical ansatz of the nonrelativistic limit of the Poincare group inherits a group theoretical meaning, in terms of generalized contractions of Lie algebras.

\section{BEHAVIOR OF CASIMIR OPERATORS UNDER NONRELATIVISTIC LIMITS}

We will now consider the nonrelativistic limit of the Casimir operators.

The Casimir operators of the Poincaré group are well known ${ }^{11}$ and can be chosen as

$$
\begin{gathered}
C_{2}^{P}=H^{2}-P_{i} P_{i}, \\
C_{4}^{P}=H^{2} J_{i} J_{i}-\left(P_{i} P_{i}\right)\left(K_{P j} K_{P j}\right)+\left(J_{i} P_{i}\right)^{2}-\left(P_{i} K_{P i}\right)^{2}-2 H J_{k} \varepsilon^{i j k} P_{i} K_{P j}
\end{gathered}
$$

over the kinematical basis. Their physical interpretation can be obtained in the center of mass system, where $P_{i}=0$ and $H=E=m_{0} \cdot{ }^{15}$ It follows that

$$
C_{2}^{P}=m_{0}^{2}, \quad C_{4}^{P}=m_{0}^{2} J_{i} J_{i}
$$

and we conclude that the two Casimir operators define the mass squared and the spin squared, where the mass $m_{0}$ will be considered as the internal energy in the one particle case. As observed earlier, we postulate that these operators actualize. This postulate is based on experimental grounds, since the mass and the spin of elementary particles are always well defined in the preparation and measurement stages of a scattering process. 
On the other hand, the Casimir operators of the trivially extended Poincare group $I S O(1,3)$ $\times\langle M\rangle$, over the basis $\left\{J_{i}, P_{i}, K_{P i}, \bar{H}, M\right\}$, are given by (see Appendix)

$$
\begin{gathered}
C_{1}^{P E}=M, \quad C_{2}^{P E}=-\left(P_{i} P_{i}\right)+\bar{H}^{2}+M^{2}+2 \bar{H} M \\
C_{4}^{P E}=\left(J_{i} J_{i}\right)(\bar{H}+M)^{2}-\left(J_{i} P_{i}\right)^{2}-\left(P_{i} P_{i}\right)\left(K_{P i} K_{P i}\right)+\left(P_{i} K_{P i}\right)^{2}-2(\bar{H}+M) \varepsilon_{i j k} J_{k} P_{i} K_{P j} .
\end{gathered}
$$

We observe that the change in basis in $I S O(1,3) \times\langle M\rangle$ explicitly introduces $\bar{H}$ and $M$ into the noncentral Casimir operator in a nontrivial way. In the center of mass system $\left(P_{i}=0\right)$ these operators simplify to

$$
C_{1}^{P E}=m_{0}, \quad C_{2}^{P E}=m_{0}^{2}, \quad C_{4}^{P E}=m_{0}^{2} J_{i} J^{i} .
$$

As in the previous case, when $P_{i}=0$, the second Casimir operator simplifies to $C_{2}^{P E}=(\bar{H}+M)^{2}$ $=H^{2}$, showing that the nonrelativistic limit of the Hamiltonian is $H \rightarrow M$ (see Sec. IV A). It is easily seen that the Casimir operators of the last equation coincide with the operators of Eq. (11), with the same interpretation, to which $C_{1}^{P E}$ is added, the latter simply corresponds to the mass.

Proceeding in analogous manner, it is straightforward to see that the Casimir operators of the mass central-extended Galilei group are

$$
\begin{gathered}
C_{1}^{G}=M=m_{0}, \\
C_{2}^{G}=M E-\frac{P^{2}}{2}=M\left(H-\frac{P^{2}}{2 m_{0}}\right)=m_{0} W, \\
C_{4}^{G}=M^{2} J_{i} J_{i}-\left(P_{i} P_{i}\right)\left(K_{G i} K_{G i}\right)+\left(P_{i} K_{i}\right)^{2}-2 M J_{k} \varepsilon^{i j k} P_{i} K_{G j},
\end{gathered}
$$

where $W$ is the internal energy. In the center of mass system these operators have the form

$$
C_{1}^{G}=m_{0}, \quad C_{2}^{G}=m_{0}^{2}, \quad C_{4}^{G}=m_{0}^{2} J_{i} J^{i} .
$$

They therefore inherit the interpretation of proper mass, the proper mass squared, and the spin squared by the mass squared, coinciding with the Casimir of Eq. (13).

\section{A. The limits among the Poincaré and Galilei groups}

Following the previous section, the nonrelativistic limit of the Casimir operators of the Poincaré group, Eq. (11), onto the Casimir operators of the extended Galilei group (15) can be obtained in the following way.

(1) $C_{2}^{P}=H^{2}-P_{i} P_{i}=m_{0}^{2}$ is the proper mass squared, and, in the one particle case $W^{2}$, namely, $C_{1}^{G 2}$ or $C_{2}^{G}$ [cf. Eq. (15)].

(2) If we restore the $c$ factors in Eq. (10) we have that

$$
C_{4}^{P} \sim c^{-4} H^{2} J_{i} J_{i}-\left(P_{i} P_{i}\right)\left(K_{P i} K_{P i}\right)-\left(m_{0} J_{i} P_{i}\right)^{2}+\left(P_{i} K_{P i}\right)^{2}-2 c^{-2} H J_{k} \varepsilon^{i j k} P_{i} K_{P j} .
$$

(3) The term $\left(m_{0} J_{i} \beta_{i}\right)^{2}$ becomes much smaller than the remaining ones if $\beta_{i} \ll 1$. Therefore $C_{4}^{P}$ $\rightarrow C_{4}^{G}$ of Eq. (14).

We conclude that, in the center of mass system, the following limits hold:

$$
C_{2}^{P} \rightarrow C_{2}^{G}=C_{1}^{G 2}, \quad C_{4}^{P} \rightarrow C_{4}^{G} .
$$

So, in this case the two Casimir operators of the Poincare group $\left(C_{2}^{P}, C_{4}^{P}\right)$ go to the three Casimir operators of the extended Galilei group $\left(C_{1}^{G}, C_{2}^{G}, C_{4}^{G}\right)$. This anomaly, two operators that go to three, is logically originated by the fact that we go from a nonextended group to a extended one. 
Nevertheless, we could also say that somehow it is usual that two physical nonrelativistic entities become just one relativistic entity, e.g., space and time become space-time. This fact would explain why the two Galilei Casimir operators $C_{1}^{G}, C_{2}^{G}$ [but really one since $C_{2}^{G}=\left(C_{1}^{G}\right)^{2}$ ] become just one relativistic Poincaré Casimir $C_{2}^{P}$.

We now proceed to justify the preceding apparent anomaly by pure group theoretical arguments, contracting the Casimir operators of the trivially extended Poincare group onto those of the extended Galilei group. Using the rescaled basis (9) and expressing the Casimir operators, we obtain that

$$
\begin{gathered}
\widetilde{C}_{1}^{P E}=\varepsilon^{-2} M^{\prime}, \quad \widetilde{C}_{2}^{P E}=-\varepsilon^{-2}\left(P_{i} P_{i}\right)+\bar{H}^{2}+\varepsilon^{-4} M^{2}+2 \varepsilon^{-2} \bar{H} M, \\
\widetilde{C}_{4}^{P E}=\left(J_{i} J_{i}\right)\left(\bar{H}+\varepsilon^{-2} M\right)^{2}-\varepsilon^{-2}\left(J_{i} P_{i}\right)^{2}-\varepsilon^{-4}\left(P_{i} P_{i}\right)\left(K_{P i} K_{P i}\right)+\varepsilon^{-4}\left(P_{i} K_{P i}\right)^{2}-2 \varepsilon^{-2}(\bar{H} \\
\left.+\varepsilon^{-2} M\right) \varepsilon_{i j k} J_{k} P_{i} K_{P j} .
\end{gathered}
$$

The contracted Casimir operators are recovered, by the usual procedure, for $\varepsilon=0$,

$$
\begin{gathered}
\hat{C}_{1}^{P E}=\lim _{\varepsilon \rightarrow 0} \varepsilon^{2} \widetilde{C}_{1}^{P E}=M^{\prime}, \quad \hat{C}_{2}^{P E}=\lim _{\varepsilon \rightarrow 0} \varepsilon^{4} \widetilde{C}_{2}^{P E}=M^{\prime 2}, \\
\hat{C}_{4}^{P E}=\lim _{\varepsilon \rightarrow 0} \varepsilon^{4} \widetilde{C}_{4}^{P E}=-\left(P_{i} P_{i}\right)\left(K_{P i} K_{P i}\right)+\left(P_{i} K_{P i}\right)^{2}+\left(J_{i} J_{i}\right) M^{2}-2 M \varepsilon_{i j k} J_{k} P_{i} K_{P j} .
\end{gathered}
$$

From this equation and (14), it follows at once that the nonrelativistic limit is simply

$$
\hat{C}_{1}^{P E}=C_{1}^{G}, \quad \hat{C}_{2}^{P E}=C_{2}^{G}, \quad \hat{C}_{4}^{P E}=C_{4}^{G} .
$$

Now we have three Casimir operators that go to three operators because now we go from an extended group to an extended group.

So, there is a clearly better behavior if we proceed with an extended Poincare group for QFT, even if the extension is trivial. In fact, from Wightman axiom A we know that, in QFT, we deal with a Hilbert space composed by normalized "rays," which are invariant under a phase transformation (see Ref. 16, p. 58, or Ref. 9, p. 67). This kind of groups deserves projective representations as in the extended groups above.

Then, from the nonrelativistic limit of the Poincare Casimir operators that do actualize according to our postulate in the introduction (and well known physical facts), we deduce that, in the relativistic limit, the Galilei Casimir operators also actualize, as proven in Ref. 7 based on the postulates of MHI.

\section{THE MANY PARTICLE CASE FOR THE IN AND OUT STAGES}

It is well known that in nonrelativistic mechanics there are continuous systems that cannot be conveniently modeled by a set of particles (solid, waves, fluid, etc.). On the contrary, in QFT, at the in and out stages, all systems can be modeled as a collection of $N$ particles (for simplicity we will only consider sets consisting of a unique type of elementary particles) and therefore the relevant group can be identified with the tensor product of $N$-copies of the Poincaré group. It turns out that the representations of this tensor product are expressible as products of corresponding representations of the factor groups, so that these representations are labeled by $N$ and the two (or three in the extended case) Casimir operators of the Poincare group. In this sense, the particle number operator $N$ becomes an extra Casimir operator to be taken into account.

Let the particle type be labeled by $C_{2}^{P}$ and $C_{4}^{P}$ or $C_{1}^{P E}, C_{2}^{P E}$, and $C_{4}^{P E}$ (as in Sec. V). Since a $n$ particle state is given by

$$
|n\rangle=|1\rangle \otimes|1\rangle \otimes \cdots|1\rangle=\left(a^{\dagger} \otimes a^{\dagger} \otimes \cdots a^{\dagger}\right)|0\rangle=\left(a^{\dagger}\right)^{n}|0\rangle,
$$

where $a^{\dagger}$ is the creation operator of the particle we are considering, and 


$$
N|n\rangle=n|n\rangle,
$$

the particle number is easily seen to be additive. Now we can combine the already known Casimir operators [those of Eq. (11)] and define new operators for $N$-particles with the same mass and spin,

$$
\text { Mass }=\left(G_{2}^{P}\right)^{1 / 2} N ; \quad \text { Spin }=G_{4}^{P}, \quad \text { Particle Number }=N .
$$

Based on experimental facts, we state that these operators do actualize.

For the sake of completeness we could consider also the nonrelativistic limit of $N$ that would be the $N$ of nonrelativistic quantum mechanics, since the first is obtained from the $a^{\dagger}$ that creates the relativistic particles, and the second obtained by its nonrelativistic limit, the $a^{\dagger}$ that corresponds to nonrelativistic particles. Of course, in the interaction stage we cannot consider the particle number operator. We can only consider the Casimir operators of total mass, total internal energy, and total spin, that do actualize.

\section{TOWARD A MHI FOR QFT}

In this section, in order to see how the previous results can be used, we will anticipate some reasonings and consequences of a future possible extension of MHI to QFT. We remember that the MHI belongs to the modal family of interpretation, i.e., it is a realist, noncollapse approach according to which the quantum state describes the possible properties of the system but not their actual properties (see Refs. 4 and 5). In this interpretation the Hamiltonian is essential for the definition of the quantum systems and in the selection of its definite-valued observables that may actualize, precisely, for MHI, they are observables that commute with the Hamiltonian. In Ref. 7 we show that the rule of definite-value ascription that selects a set of definite-valued observables must be unaltered under the Galilean transformations. Then, since the Casimir operators of the Galilean group are invariant under all the transformations of the group, it is proposed that the actualization rule may be reformulated in terms of these invariant operators. Moreover, in Secs. II-IV, we have shown that the Casimir operators have well behaved limits of the Casimir operators of the extended Poincare group. Then it is reasonable to postulate that the Casimir operators of this extended Poincare group actualize in QFT, which is of course, universally admitted since this Casimir labels the representation of the group and therefore the type of particles. But this is not the whole history since in QFT momenta actualize in the in and out stages of the scattering theory. So in this section, in order to verify these ideas about actualization in the simplest case, we study the three stages of the scattering process of QFT, the only arena where this theory has an experimental verification.

\section{A. In all three stages}

Which observables actualize in QFT? As we have explained, $M, C_{2}^{P E}$, and $C_{4}^{P E}$ constitute the most natural choice for the three stages of a QFT scattering experiment (and we know that they have a good nonrelativistic limit to the ordinary nonrelativistic quantum mechanics). The nonrelativistic limit of this hypothetical choice yields the nonrelativistic MHI developed progressively in Refs. 4, 5, and 7.

\section{B. In the in and out stages}

Let us now consider the particular case of the in and out stages, where experimentally we know that linear momenta are determined. Then we must also explain the actualization of the components of the linear momentum, and it could be possible that an additional postulate would be required. Actually this is not the case because in the preparation and measurement periods new characters appear, namely, the preparation and measurement apparatuses that essentially define the momenta, in fact,

(1) the preparation apparatus prepares a ray of particles with a well defined momentum; 
(2) in the measurement apparatus as (e.g., in a fog or bubble chamber) the momenta are well defined (e.g. by trajectories in the chamber), since their unique purpose is precisely to measure these momenta.

Then in principle in these three stages momenta are good candidates to actualize. But let us try to deduce this conclusion directly from the postulates of nonrelativistic MHI, making the nonrelativistic limit.

\section{Nonrelativistic limit}

In fact, to complete the panorama, we only need to consider the nonrelativistic limit of the preparation and measurement processes (via nonunitary evolutions) that begins and end in equilibrium states, related to low velocities (which necessarily appears in these processes and correspond to the nonrelativistic case). This fact enables us to use the Galilei group and its main features in the nonrelativistic MHI.

\section{Preparation stage}

(1) Theoretically, if we want to prepare an arbitrary state, we must begin with a ground state and then accelerate it, using an external system, from this initial state to the final state we want to reach (see, for instance, Ref. 9, Chap. 8).

(2) The facts are the same in practice; particles initially at low velocities must be accelerated.

\section{Measurement stage}

Somehow you have the inverted process of the preparation stage.

(1) Theoretically we must detect the trajectories of the outgoing particles. Two main theoretical ways for this purpose are given.

(a) We must introduce an environment, and then we have a composite system formed by the elementary particle and the fog (or the bubbles) chamber. Therefore we can explain the particle trajectory "à la Mott" "17 (or using the Bohr-Oppenheimer method, already considered in Refs. 4 and 5, since fog or the bubbles nuclei are larger than the scattered particles and therefore are fixed).

(b) According to the procedure developed in Ref. 18 using the destructive interference produced by the Hamiltonian evolution and showing how classical trajectories appear. In all these cases, we necessarily reach equilibrium, therefore previously we have low velocities that allow to use the nonrelativistic case of MHI.

(2) In practice, materializing these theoretical structures either with a fog or bubble chamber or with a detector (Geiger counter, photographic plate, etc.), we also have an irreversible and nonunitary process to reach equilibrium (general or partial but always with velocities small enough to allow the recording of the results).

So, either in the preparation or the measurement period, we have low velocities and we can use (nonrelativistic) MHI. But, the microscopical objects, introduced by the preparation and measurement apparatuses, necessarily produce some inhomogeneity and anisotropy in the total Hamiltonian (scattered particles and apparatuses), and therefore they break their eventual symmetries. We then arrive to the case of MHI (Refs. 4 and 5), where the relevant Hamiltonian has few or no symmetries. Then, there is a large number of constants of motion that introduce relevant indices in the energy spectrum, and, according to MHI, all these constants of motion actualize, among them the momenta $P_{i}$. Moreover, according to Refs. 18 and 19 also well defined classical trajectories appear. Since these trajectories are linear, they are defined by the corresponding momenta $P_{i}$, and this is a feature that proves that momenta do actualize.

In conclusion, in the relativistic case of QFT, based in our experience in the nonrelativistic one and in obvious physical facts, we can postulate that the Casimir operators do actualize, namely, 
$C_{1}^{P E}, C_{2}^{P E}$, and $C_{4}^{P E}$, where, e.g., $C_{1}^{P E}$ is the mass of the proper particle $m_{0}$ in the one particle case, and this mass multiplied by the particle number $N$ is the total mass of the system in the many particles case, in the in and out stages. Then the operators of Eq. (17) are those that actualize. Finally, a possible explanation of the actualization of linear momentum is given in this section.

This is just a sketch. We will follow this research hoping to find more precise results.

\section{CONCLUSION}

We have presented the nonrelativistic limit of the trivially extended Poincare group: the extended Galilei group. We have shown that the corresponding Casimir operators obey the same limit. Finally, we have also presented an example where this limit can be used: the nonrelativistic limit of a possible interpretation of QFT that turns out to be one of the possible interpretations of quantum mechanics, MHI. This fact gives, at least, a correct limit to our candidate interpretation for QFT. Moreover, in this way, we can see how physical actualization changes according both to the theory and the model considered and therefore may be we have found the base for a modal interpretation of QFT.

\section{ACKNOWLEDGMENTS}

The three authors are extremely grateful to the referee, whose relevant observations have greatly improved the final version of this paper. The third author acknowledges partial financial support from the Research Project No. MTM2006-09152 of the MICINN.

\section{APPENDIX: CASIMIR INVARIANTS IN OF ISO $(1,3) \oplus\langle M\rangle$}

The Casimir invariants can be computed using an analytical approach based on the realization of Lie algebras in terms of differential operators. ${ }^{20}$ For the extended Poincare $\operatorname{ISO}(1,3) \oplus\langle M\rangle$, the explicit system of partial differential equations obtained by this procedure is given by

$$
\begin{aligned}
& \hat{J}_{1} F=j_{3} \frac{\partial F}{\partial j_{2}}-j_{2} \frac{\partial F}{\partial j_{3}}+p_{3} \frac{\partial F}{\partial p_{2}}-p_{2} \frac{\partial F}{\partial p_{3}}+k_{3} \frac{\partial F}{\partial k_{2}}-k_{2} \frac{\partial F}{\partial k_{3}}=0, \\
& \hat{J}_{2} F=-j_{3} \frac{\partial F}{\partial j_{1}}+j_{1} \frac{\partial F}{\partial j_{3}}-p_{3} \frac{\partial F}{\partial p_{1}}+p_{1} \frac{\partial F}{\partial p_{3}}-k_{3} \frac{\partial F}{\partial k_{1}}-k_{1} \frac{\partial F}{\partial k_{3}}=0, \\
& \hat{J}_{3} F=j_{2} \frac{\partial F}{\partial j_{1}}-j_{1} \frac{\partial F}{\partial j_{2}}+p_{2} \frac{\partial F}{\partial p_{1}}-p_{1} \frac{\partial F}{\partial p_{2}}+k_{2} \frac{\partial F}{\partial k_{1}}-k_{1} \frac{\partial F}{\partial k_{2}}=0, \\
& \hat{P}_{1} F=p_{3} \frac{\partial F}{\partial j_{2}}-p_{2} \frac{\partial F}{\partial j_{3}}-(\bar{h}+m) \frac{\partial F}{\partial k_{1}}=0, \\
& \hat{P}_{2} F=-p_{3} \frac{\partial F}{\partial j_{1}}+p_{1} \frac{\partial F}{\partial j_{3}}-(\bar{h}+m) \frac{\partial F}{\partial k_{2}}=0, \\
& \hat{P}_{3} F=p_{2} \frac{\partial F}{\partial j_{1}}-p_{1} \frac{\partial F}{\partial j_{2}}-(\bar{h}+m) \frac{\partial F}{\partial k_{3}}=0, \\
& \hat{K}_{1} F=k_{3} \frac{\partial F}{\partial j_{2}}-k_{2} \frac{\partial F}{\partial j_{3}}+(\bar{h}+m) \frac{\partial F}{\partial p_{1}}-j_{3} \frac{\partial F}{\partial k_{2}}+j_{2} \frac{\partial F}{\partial k_{3}}+p_{1} \frac{\partial F}{\partial \bar{h}}=0,
\end{aligned}
$$




$$
\begin{aligned}
& \hat{K}_{2} F=-k_{3} \frac{\partial F}{\partial j_{1}}+k_{1} \frac{\partial F}{\partial j_{3}}+(\bar{h}+m) \frac{\partial F}{\partial p_{2}}+j_{3} \frac{\partial F}{\partial k_{1}}-j_{1} \frac{\partial F}{\partial k_{3}}+p_{2} \frac{\partial F}{\partial \bar{h}}=0 \\
& \hat{K}_{3} F=k_{2} \frac{\partial F}{\partial j_{1}}-k_{1} \frac{\partial F}{\partial j_{2}}+(\bar{h}+m) \frac{\partial F}{\partial p_{3}}-j_{2} \frac{\partial F}{\partial k_{1}}+j_{1} \frac{\partial F}{\partial k_{2}}+p_{3} \frac{\partial F}{\partial \bar{h}}=0, \\
& \hat{\bar{H}} F=p_{1} \frac{\partial F}{\partial k_{1}}+p_{2} \frac{\partial F}{\partial k_{2}}+p_{3} \frac{\partial F}{\partial k_{3}}=0 \\
& \hat{m} F=0 .
\end{aligned}
$$

Here $\{j, p, j, \bar{h}, m\}$ denote the coordinates of a basis dual to the generators of the basis $\{J, P, K, \bar{H}, M\}$.

${ }^{1}$ B. C. Van Fraassen, A Formal Approach to Philosophy of Science, Paradigms and Paradoxes: The Philosophical Challenge of the Quantum Domain (University of Pittsburgh Press, Pittsburgh, 1972), pp. 303-366.

${ }^{2}$ S. Kochen, Symposium on the Foundations of Modern Physics (World Scientific, Singapore, 1985), pp. 151-169.

${ }^{3}$ J. Bub, Interpreting the Quantum World (Cambridge University Press, Cambridge, 1997).

${ }^{4}$ O. Lombardi and M. Castagnino, Stud. Hist. Philos. Mod. Phys 39, 380 (2008).

${ }^{5}$ M. Castagnino and O. Lombardi, J. Phys.: Conf. Ser. 128, 012014 (2008).

${ }^{6}$ N. D. Mermin, Pramana 51, 549 (1998).

${ }^{7}$ J. S. Ardenghi, M. Castagnino, O. Lombardi, Found. Phys. (submitted).

${ }^{8} \mathrm{H}$. Weyl, The Theory of Groups and Quantum Mechanics (Dover, New York, 1931).

${ }^{9}$ L. Ballentine, Quantum Mechanics, a Modern Development (World Scientific, Singapore, 1998).

${ }^{10}$ Generators $J_{i}$ define the $S O(3)$ group and generators $P_{i}, J_{i}$, define the $I S O(3)$ group: the inhomogeneous rotation group in three dimensions. Actually (5) is the largest subgroup that remains invariant by the Inönü-Wigner contraction of the Poincaré on the Galilei group.

${ }^{11}$ M. Bacry and J.-M. Levy Léblond, J. Math. Phys. 9, 1605 (1968).

${ }_{12}^{12}$ J. F. Cariñena, M. A. del Olmo, and M. Santander, J. Phys. A 14, 1 (1981).

${ }^{13}$ By a trivial extension of a Lie algebra $\mathfrak{g}$ we mean the direct sum $\mathfrak{g} \oplus M$, where $M$ is an additional commuting generator.

${ }^{14}$ See also in Ref. 21 for a $c=1$ deduction.

${ }^{15}$ For the same reasons explained in Ref. 7, where the transformation $H \rightarrow W$ is introduced, and the nonrelativistic case analyzed.

${ }^{16}$ R. Haag, Local Quantum Physics (Springer-Verlag, Berlin, 1993).

${ }^{17}$ N. F. Mott, Proc. R. Soc. London, Ser. A 126, 79 (1929).

${ }^{18}$ M. Castagnino and R. Laura, Int. J. Theor. Phys. 39, 1767 (2000); Phys. Rev. A 62, 022107 (2000).

${ }^{19}$ M. Castagnino and O. Lombardi, Phys. Rev. A 72, 012102 (2005); Stud. Hist. Philos. Mod. Phys. 35, 73 (2004).

${ }^{20}$ E. G. Beltrametti and A. Blasi, Phys. Lett. 20, 62 (1966).

${ }^{21}$ J.-M. Levy Léblond, Ann. Inst. Henri Poincare, Sect. A 3, 1 (1965). 